\title{
Impact of frailty on hospital adverse outcomes in elderly admitted with acute coronary syndrome
}

\author{
A Ponciano, F Montenegro Sá, F Saraiva, F Soares, J Leite, J Morais \\ Centro Hospitalar de Leiria, Leiria, Portugal
}

\begin{abstract}
Background: Frailty represents a complex clinical syndrome of decreased physiological reserve and increased vulnerability to stressors. Little is known regarding interaction between frailty status and outcomes in patients with acute coronary syndrome. Our study aims to assess the prevalence of frailty and its impact on in-hospital adverse outcomes of patients aged $\geq 75$ years admitted for acute coronary syndromes.

Methods: In this retrospective, single centre, observational study were included patients aged $\geq 75$ years admitted for acute coronary syndromes between January 2011 and December 2015. Frailty was assessed using the Fried criteria. The primary endpoint was all-cause in-hospital mortality. Secondary endpoints included the occurrence of re-infarction, stroke and major bleeding.

Results: 0 the 502 patients included, 126 were classified as frail. These patients were older (mean age $78 \pm 5.5$ vs. $76.2 \pm 5.5$ years; $p=0.020$ ), more often male (68.3\%) and had a higher risk profile according to GRACE (151.4 \pm 18.8 vs. $132.1 \pm 16.8 ; p<0.001)$, TIMl $(4.3 \pm 1$ vs. $3.1 \pm 1 ; p<0.001)$ and CRUSADE (34.6 \pm 9.4 vs. $25.8 \pm 9.5 ; p<0.001)$ scores at admission. All-cause in-hospital mortality was significantly more frequent in frail patients $(11.9 \%$ vs. $5.6 \% ; p<0.001)$, as well as re-infarction (7.4\% vs. $4.8 \%$; $p<0.001)$, stroke ( $8.7 \%$ vs. $0.5 \% ; p=0.002)$ and major bleeding $(7.9 \%$ vs. $1.6 \% ; p=0.002)$. On multivariate analysis, frailty remained independently associated with the primary endpoint (OR 5.63, [2.05-10.35]; $p<0.001)$.

Conclusion: Frailty, identified by Fried criteria, is frequent in elderly patients with acute coronary syndromes, and it is an independent prognostic predictor for in-hospital mortality.

Keywords: acute coronary syndrome; elderly; frailty; prognosis.

Palabras clave: Síndrome coronario agudo. Anciano. Fragilidad. Pronóstico.
\end{abstract}

\section{INTRODUCTION}

Cardiovascular disease (CVD) remains the main cause of morbidity and mortality in the developed countries and within the last decades there was a remarkable shift in the burden of CVD towards older people., ${ }^{1,2}$

Aging of population and high incidence of acute coronary syndromes (ACS) in the elderly point towards a marked increase in the number of elderly patients admitted for ACS.

Globally, around $30 \%$ of ACS patients are over 75 yearsold. ${ }^{3}$ The risk of adverse cardiovascular events after ACS increases with age. ${ }^{4-7} \mathrm{An}$ appropriate Risk stratification is of paramount importance in order to plan medical care and may improve prognosis in older population. ${ }^{8}$

Despite the higher risk, elderly patients are frequently underrepresented in clinical trials responsible for a clear gap in guidelines. ${ }^{9-11}$

Frailty is a biological syndrome reflecting a state of decreased physiological reserve and vulnerability to stressors such as an acute myocardial infarction. ${ }^{12}$ It is considered to be highly prevalent with increasing age and conveys a higher risk of adverse outcomes. ${ }^{13}$

Several diagnostic tools have been developed to measure frailty, either by questionnaires or simple measures, with no clear consensus on which is the best.

The Fried scale encompasses slowness, weakness, low physical activity, exhaustion, unintentional weight loss and it is considered a more reliable predictor for adverse outcome than chronological age. ${ }^{14}$ Although there are well defined pathways for ACS management, data applicability to elderly patients is questionable, since most randomized clinical trials often exclude such patients. ${ }^{15}$

The aim of the present study is to assess the prevalence of frailty and its impact on in-hospital adverse outcomes of patients aged $\geq 75$ years admitted for acute coronary syndromes.

\section{MATERIAL AND METHODS}

A retrospective, observational single-center analysis was performed in coronary care unit in a non-tertiary care hospital in Portugal from January 2011 to December 2015 and included patients aged $\geq 75$ years admitted for acute coronary syndrome who underwent percutaneous coronary intervention. ${ }^{16}$

Exclusion criteria included severe cognitive impairment, cardiogenic shock or prolonged electrical/haemodynamic instability at presentation, and limited life expectancy due to neoplasic disorders.

In every patient, baseline clinical characteristics including demographic and clinical data, comorbidities and laboratory data were collected. Prognostic ACS indexes were also analyzed, namely Global Registry of Acute Coronary Events (GRACE), Thrombolysis in Myocardial Infarction (TIMI), Can Rapid risk stratification of Unstable angina patients Suppress ADverse outcomes with Early implementation of the ACC/ AHA guidelines Quality Improvement Initiative (CRUSADE) and Charlson Comorbidity Index. ${ }^{17}$ 
The GRACE risk score uses eight independent predictors of death or the combined outcome of death and Ml, both in hospital and at 6 months: age, admission heart rate and systolic blood pressure, Killip-Kimball class, the initial serum creatinine concentration, cardiac arrest at admission, STsegment deviation and elevated cardiac markers. A score greater than 140 identifies high-risk patients who have greater benefit from an early invasive strategy. ${ }^{18}$

The TIMI risk score is a semi-quantitative score that includes seven variables: age $>65$ years, three or more cardiovascular risk factors, known $\geqslant 50 \%$ coronary artery stenosis, aspirin use within last 7 days, $\leqslant 24$ hours angina, $\geqslant 0.5 \mathrm{~mm}$ STsegment deviation and positive cardiac markers. TIMI punctuation predicts 14-day risk of the composite endpoint of death, $\mathrm{Ml}$ and urgent revascularisation. ${ }^{19}$

The CRUSADE bleeding score identifies eight independent predictors of major bleeding (intracranial haemorrhage, retroperitoneal bleed, haematocrit drop $\geqslant 12 \%$, or the need for red blood cells transfusion) during ACS hospitalisation: baseline haematocrit $<36 \%$, creatinine clearance, heart rate, female sex, signs of heart failure at admission, systolic blood pressure $\leqslant 110 \mathrm{mmHg}$ or $\geqslant 180 \mathrm{mmHg}$, prior vascular disease and diabetes mellitus. A score of 20 or less defines a very low risk of severe bleeding (3.1\%), whereas punctuation over 50 defines a very high risk (19.5\%). ${ }^{20}$

Frailty status evaluation was obtained within the first 48 hours of admission by a qualified physician. Frailty was assessed using the Fried criteria. The Fried scale comprises a possible range from 5 points based on the presence or absence of five frailty criteria- slowness assessed by walk speed, weakness assessed by hand grip strength and selfreported low physical activity, exhaustion, and unintentional weight loss. Three or more criteria assessed as positive defines a patient as frail. ${ }^{14,21}$

For the purpose of this study, two groups were distinguished according to Fried criteria: frail and non-frail patients.

The study protocol is according to the Declaration of Helsinki. The collection of clinical information for this study was approved by our hospital institutional review board.

All patients or their guardians provided informed consent for participation.

Endpoints: The primary endpoint was all-cause in-hospital mortality. Secondary endpoints included: the occurrence of re-infarction; stroke; major bleeding, defined as a drop of $\geq 3 \mathrm{~g} / \mathrm{dl}$ of hemoglobin attributable to blood loss or the need for surgery or any transfusions during hospitalization.

\section{Statistical analysis}

Categorical variables were described as frequencies and percentages. Continuous variables were described by mean \pm standard deviation (SD). Statistical differences between groups were assessed using the $\chi 2$ test and Fisher's exact test when appropriate for categorical variables, Student's t-test for continuous variables of normal distribution or the Mann-Witney $U$ test for non-normal distribution. A two-sided $\mathrm{P}<0.05$ was considered significant. Multiple logistic regression analysis was used to evaluate the possible association between frailty phenotype and predefined primary and secondary endpoints. We estimated adjusted Odds Ratio and 95\% confidence intervals (Cl).

The model was adjusted for previously selected variables, including validated prognostic indexes such as the GRACE score, the CRUSADE bleeding score, the TIMI score, maximum in-hospital creatinine level, culprit lesion revascularization and complete revascularization (defined as percutaneous treatment of all severe coronary stenosis). These variables are widely used and recognized as prognostic variables. Previous selection of recognized prognostic values was done to increase the external validity of this study.

All the statistical analyses were performed using statistical package for social sciences V.22.0, statistical software (SPSS, Chicago, Illinois, USA).

\section{RESULTS}

Of the 502 patients included, $25.1 \%(n=126)$ were classified as frail. The mean age was 77 years, and $31.1 \%$ were women.

The prevalence of diabetes mellitus was $30.9 \%$, and $75 \%$ had hypertension. Baseline characteristics according to frailty status are presented in Table 1. Frail patients were older ( $78 \pm 5.5$ vs $76+5,5 ; p=0.020)$, had higher comorbidity features with a higher score on the Charlson Comorbidity Index and dyslipidemia was also more frequent in this group ( $57.1 \%$ vs $44.9 \% ; p=0.020$ ).

At admission, frail population presented with higher risk profile according to the GRACE $(151.4 \pm 18.8$ vs. $132.1 \pm 16.8 ; p<0.001)$, TIMI $(4.3 \pm 1$ vs. $3.1 \pm 1 ; p<0.001)$ and CRUSADE $(34.6 \pm 9.4$ vs. $25.8 \pm 9.5 ; p<0.001)$ scores.

The primary endpoint of in-hospital all-cause mortality occurred more frequently in frail patients $(11.9 \%$ vs. $9.6 \% ; p<0.001)$, as well as secondary endpoints: reinfarction $(7.1 \%$ vs. $4.8 \%$; $p<0.001)$; stroke $(8.7 \%$ vs. $0.5 \% ; p=0.002)$; and major bleeding $(7.9 \%$ vs. $1.6 \%$; $\mathrm{p}=0.002)$. On multivariate analysis, after adjustment for GRACE and CRUSADE admission values, maximum inhospital creatinine level, culprit lesion revascularization and complete revascularization, frailty status was an independent prognostic marker for inpatient all-cause mortality (OR 5.63, [2.05-10.35]; $p<0.001)$.

\section{DISCUSSION}

To our knowledge this is the first Portuguese analysis of frailty regarding acute coronary syndrome giving insight of a real-life coronary unit experience.

The management of older ACS patients is challenging because not only age but also multiple factors contribute to adverse outcomes and therefore must be taken in consideration. 
Table 1. Baseline characteristics and overall risk estimation

\begin{tabular}{|c|c|c|c|}
\hline & Frail $(n=126)$ & Non-frail( $(n=376)$ & $P$ value \\
\hline Age (mean $\pm S D ; y e a r s)$ & $78 \pm 5.5$ & $76 \pm 5.5$ & 0.020 \\
\hline Gender: Male (\%) & $86(68.3 \%)$ & $260(69.1 \%)$ & 0.800 \\
\hline Arterial Hypertension (\%) & $95(75.4 \%)$ & $281(74.7 \%)$ & 0.880 \\
\hline Diabetes Mellitus (\%) & $42(33 \%)$ & $113(30.1 \%)$ & 0.500 \\
\hline Dyslipidaemia (\%) & $72(57.1 \%)$ & $169(44.9 \%)$ & 0.020 \\
\hline Smokers (\%) & $11(8.7 \%)$ & $58(15.4 \%)$ & 0.060 \\
\hline Renal impairment (\%) & $9(7.1 \%)$ & $17(4.5 \%)$ & 0.250 \\
\hline CICreatinine $>50 \mathrm{~mL} / \mathrm{min}$ & $6(4.8 \%)$ & $9(2.4 \%)$ & 0.170 \\
\hline CICreatinine $<50 \mathrm{~mL} / \mathrm{min}$ & $3(2.4 \%)$ & $8(2.2 \%)$ & 0.890 \\
\hline Previous heart failure (\%) & $4(3.2 \%)$ & $6(1.6 \%)$ & 0.260 \\
\hline Previous myocardial infarction (\%) & $16(12.7 \%)$ & $54(14.4 \%)$ & 0.640 \\
\hline Previous stroke (\%) & $11(8.7 \%)$ & $30(8.0 \%)$ & 0.790 \\
\hline GRACE score (mean \pm SD) & $151.4 \pm 18.8$ & $132.1 \pm 16.8$ & $<0.001$ \\
\hline TIMI score (mean $\pm S D)$ & $4.3 \pm 1.0$ & $3.1 \pm 1$ & $<0.001$ \\
\hline CRUSADE score (mean $\pm S D)$ & $34.6 \pm 9.4$ & $25.8 \pm 9.5$ & $<0.001$ \\
\hline Chalrson Comorbidity Index & $7.9 \pm 2.3$ & $6.4 \pm 1.7$ & $<0.001$ \\
\hline
\end{tabular}

Frailty phenotype confers an age independent risk for adverse outcomes.

In this study, more than a quarter of the population was frail. Frail patients were slightly older and presented an increased burden of disease, with higher prevalence of comorbidities. They also presented a higher risk according to the GRACE, TIMI and CRUSADE scores, hence higher probability for adverse events. ${ }^{22}$

The current study demonstrated that in patients with ACS aged $\geq 75$ years, frailty is an important prognostic risk factor for in-hospital mortality and major cardiovascular events. This prognostic value is independent of age and risk scores. These findings are in accordance with multinational published registries which support a positive correlation between frailty and poor outcomes in ACS among elderly patients with high-risk ACS. ${ }^{23-25}$

Patients included in TaRgeted platelet Inhibition to cLarify the Optimal strateGy to medicallY manage Acute Coronary Syndromes (TRILOGY ACS) trial were classified as frail, prefrail and non-frail based on the Fried criteria as used in our study. Using this self-reported based method, only $4.7 \%$ of included patients were classified as frail. After adjustment for characteristics and GRACE score, frailty was significantly associated with the combination of death, myocardial infarction and stroke in this population of non-invasively managed ACS patients. ${ }^{26}$

Some limitations to this study should be noted. This is an observational, single centre study with a small number of patients and retrospectively collected data. The assessment of frailty was only collected at baseline and was not repeated later to assess change. However, it has the advantage of representing real life clinical practice. Some answers to the Fried index were self-reported by patients, which may allow some bias by the subjectivity of each individual. Although self-reporting is prone to confounding factors, several studies have reported the value of self-reporting of frailty. ${ }^{4} \mathrm{We}$ found that frail patients had a higher prevalence of diabetes, renal impairment, hypertension and previous cardiovascular events, although not statistically significant, due to relatively small sample in the present study. It would be of interest to perform a longer follow-up study with prospectively collected data and a larger sample in order to extend these observations. Frailty, identified by Fried criteria, is frequent in elderly patients with acute coronary syndromes, and it is an independent prognostic predictor for in-hospital mortality. Frailty is frequent in over 75 year's old patients with ACS. It is a predictor for adverse events, being a more reliable prognostic factor than chronological age. This suggests that 
age by itself should not be our first and main prognostic evaluation for this patients group. Treatment approach should be individualized, including also a functional status evaluation.

\section{REFERENCES}

1. Singh $M$, Stewart $R$, White $H$. Importance of frailty in patients with cardiovascular disease. Eur Heart J 2014; 35: 1726-1731.

2. Graham MM, Galbraith PD, O'Neill D, et al. Frailty and Outcome in Elderly Patients With Acute Coronary Syndrome. Can J Cardiol 2013; 29: 1610-1615.

3. Malkin CJ, Prakash R, Chew DP. The impact of increased age on outcome from a strategy of early invasive management and revascularisation in patients with acute coronary syndromes: retrospective analysis study from the ACACIA registry. Am J CArdiol 2009; 103: 1530.

4. Newby LK, Bhapkar MV, White HD, et al. Predictors of 90-day outcome in patients stabilized after acute coronary syndromes. Eur Heart J 2003; 24:172-81.

5. Halon DA, Adawi S, Dobrecky-Mery I, et al. Importance of increasing age on the presentation and outcome of acute coronary syndromes in elderly patients. J Am Coll Cardiol 2004; 43:346-52.

6. Angeli F, Verdecchia P, Savonitto S, et al. Early invasive versus selectively invasive strategy in patients with non-ST-segment elevation acute coronary syndrome: impact of age. Catheter Cardiovasc Interv 2014;83: 686-701.

7. Dodd KS, Saczynski JS, Zhao Y, et al. Exclusion of Older Adults and Women from Recent Trails of Acute Coronary Syndromes. J Am Geriatr Soc 2011; 59:506-511.

8. Afilalo J, Alexander KP, Mack MJ, et al. Frailty assessment in the Cardiovascular Care of Older Adults. JACC 2014; 8:747-762.

9. Lee PY, Alexander KP, Hammill BG, et al. Representation of elderly persons and women in published randomized trials of acute cornoray syndromes. JAMA. 2001; 286:708-713.

10. Krumholz HM, Gross CP, Peterson ED, et al. Is there evidence of implicit exclusion criteria for elderly patients in randomized trials? Evidence from GUSTO-1 study. Eur Heart J 2003; 146:839-847.

11. Sanchis J, Bonanad C, Ruiz V, et al. Frailty and other geriatric conditions for risk stratification of older patients with acute coronary syndrome. Am Heart J 2014; 168:784-791.

12. Fried LP, Tangen CM, Walston J, et al. Frailty in Older Adults: Evidence for a Phenotype. J Gerontol A Biol Sci Med Sci 2001; 56(3):M146-56.

13. Rockwood K, Mitnitsky A. Frailty Defined by Deficit Accumulation and Geriatric Medicine Defined by Frailty. Clin Geriatr Med 2011; 27:17-26.

14. Clegg A, Young J, lliffe S, et al. Frailty in elderly people. Lancet 2013; 381:752762.

15. Alexander KP, Roe MT, Chen AY, et al. Evolution in cardiovascular care for elderly patients with non-ST-segment elevation acute coronary syndromes: results from the CRUSADE National Quality Improvemente Initiative. J Am Coll Cardiol 2005; 46:1479-1487.
16. Thygesen K, Alpert JS, Jaffe AS, et al. Third Universal definition of myocardial infarction. Eur Heart J 2012; 33: 2551-2567

17. Charlson ME, Pompei P, Ales KL, et al. A new method of classifying prognostic comorbidity in longitudinal studies: development and validation. J Chronic Dis 1987; 40:373-383

18. Granger CB, Goldberg RJ, Dabbous 0 , et al. Predictors of hospital mortality in the global registry of acute coronary events. Arch Intern Med 2003;163(19):23452553.

19. Antman EM, Cohen M, Bernink PJ. The TIMI risk score for unstable angina/non-ST elevation Ml: a method for prognostication and therapeutic decision making. JAMA 2000; 284: 835-842.

20. Subherwal S, Bach RG, Chen AY. Baseline risk of major bleeding in non-ST-segment-elevation myocardial infarction: the CRUSADE (Can Rapid risk stratification of Unstable angina patients Suppress Adverse outcomes with Early implementation of the ACC/AHA Guidelines) bleeding score. Circulation 2009; 119: 1873-1882.

21. Drey M, Pfeifer K, Sieber CC, et al. The Fried Frailty Criteria as Inclusion Criteria for a Randomized Controlled Trial: Personal Experience and Literature Review. Gerontology 2011; 57:11-18.

22. Chen X, Mao G, Leng SX. Frailty syndrome: an overview. Clin Interv Aging 2014; 9:433-441.

23. Velders MA, James SK, Libungan B, et al. Prognosis of elderly patients with ST-elevation myocardial infarction treated with primary prercutaneous coronary intervention in 2001 to 2011: a report from the Swedish Coronary Angiography and Angioplasty Registry (SCAAR) registry. Am Heart J 2014; 167: 666-673.

24. TIME investigators. Trial of invasive versus medical therapy in elderly patients with chronic symptomatic coronary-artery disease (TIME): a randomised trial. Lancet 2001; 358: 951-957.

25. Ekerstad N, Swahn E, Janzon M, et al. Frailty is independently associated with 1-year mortality for elderly patients with non-ST- segment elevation myocardial infarction. Eur J Prev Cardiol 2014; 21: 1216-1224.

26. White HD, Westerhout CM, Alexander KP, et al. Frailty is associated with worse outcomes in non-ST-segment elevation acute coronary syndromes: Insights from the TaRgeted platelet Inhibition to cLarify the Optimal strateGy to medicallY manage Acute Coronary Syndromes (TRILOGY ACS) trial. Eur Heart J. Acute Cardiovasc Care 2016; 5:2312-242. 